\title{
IMUNOISTOQUÍMICA EM OLIGODENDROGLIOMAS
}

\author{
Arlete Hilbig', Lígia Maria Barbosa-Coutinho², Gabriel Corteze Netto3, \\ Cristina Birlem Bleil${ }^{4}$, Nadima Vieira Toscani ${ }^{4}$
}

\begin{abstract}
RESUMO - Os oligodendrogliomas (OL) são tumores gliais caracterizados histologicamente pela presença de núcleo redondo e homogêneo com halo claro perinuclear. A diferenciação microscópica desses tumores com neurocitoma central, DNT e algumas vezes com ependimoma de células claras pode ser difícil. O estudo imunoistoquímico com marcadores glial e neuronal tem sido utilizado e pode auxiliar no diagnóstico diferencial. $\mathrm{O}$ objetivo do presente estudo foi determinar a diferenciação neuronal e glial por meio de técnica imunoistoquímica utilizando anticorpos de rotina em tumores com características microscópicas de OL. Foram estudados 42 pacientes com idade entre 4 e 60 anos. Dez apresentavam sinais de maior malignidade (anaplásico). Trinta e três casos (78,5\%) mostraram positividade para GFAP, sendo em 10 focal e 6 casos com expressão intensa. Doze casos $(28,5 \%)$ apresentaram positividade para NSE e/ou sinaptofisina, demonstrando alguma diferenciação neuronal, principalmente focal. Trinta e quatro casos $(80,9 \%)$ foram positivos para S-100 e três casos $(7,1 \%)$ foram positivos focalmente para NeuN. Concluimos que áreas focais de diferenciação neuronal e/ou glial podem estar presente em OL típicos e, portanto, é necessário cautela no diagnóstico diferencial em amostras pequenas de tumor. A positividade difusa para marcadores neuronais deve sugerir o diagnóstico de neurocitoma central.
\end{abstract}

PALAVRAS-CHAVE: oligodendroglioma, marcador neuronal, imunoistoquímica.

\section{Immunohistochemstry in oligodendrogliomas}

ABSTRACT - Oligodendrogliomas (OL) are neuroepithelial tumors characterized by the presence of uniformly round nuclei with a clear cytoplasm around it. These features can also be seen in central neurocytomas, DNTs and clear cell ependymomas. Immunohistochemstry with glial and neuronal markers may be helpful in diferential diagnosis. The aim of this study was to determine the glial and neuronal diffe rentiation in 42 specimes of otherwise typical OL using immunohistochemical techniques. Ten cases showed anaplastic characteristics. Thirty-th re samples (78.5\%) were positive to GFAP with few cells stained in ten cases and many positive cells in six. Twelve cases (28.5\%) were focally positive to NSE and/or synaptophysin showing n e u ronal differentiation. Thirty-four cases $(80.9 \%)$ expressed S-100. In conclusion, glial proteins may be p resent focally in $\mathrm{OL}$ due to presence of mature reactive astrocytes or transitional forms between astrocytes and oligodendrocytes. Focal areas of neuronal differentiation can also be found in typical OL. The widespread staining with neuronal marker suggests central neurocytoma, but this diagnosis should not be done with small amount of tissue.

KEY WORDS: oligodendrogliomas, neuronal markers, imunohistochemstry.

Os oligodendrogliomas (OL) são tumores gliais de baixa malignidade e crescimento lento que acometem p redominantemente a substância branca dos hemisférios cerebrais, com tendência a envolvimento cortical. São mais comuns na região fronto-temporal e rep re sentam aproximadamente $5 \%$ dos tumores intracranianos e 10 a $17 \%$ dos gliomas ${ }^{1,2}$. Nos últimos anos houve aumento expressivo no diagnóstico de OL em função da possível sensibilidade desses tumores à qui- mioterapia ${ }^{2-4}$. Além disso, a presença de componente oligodendroglial em tumores de maior grau de malignidade parece ser um fator relacionado a maior sobrevida ${ }^{5,6}$. Dessa forma, os patologistas se viram compelidos a procurar por células com núcleo pequeno e redondo com halo claro perinuclear em todos os tumores estudados. Entretanto, a diferenciação mic roscópica entre $\mathrm{OL}$, neurocitoma central e algumas vezes com ependimoma de células claras, tumor neu-

Programa de Pós-graduação em Patologia da Fundação Faculdade Federal de Ciências Médicas de Porto Alegre (FFFCMPA), Porto Alegre RS, Brasil: 'Médica Neurologista, Mestre e Doutora em Medicina:Clínica Médica pela (UFRGS). Professora Adjunta de Anatomia Humana da FFFCMPA; ${ }^{2}$ Médica Neuropatologista, Mestre e Doutora em Medicina, Clínica Médica pela UFRGS, Livre-docente e Professora Titular em Anatomia Patológica da FFFCMPA; ${ }^{3}$ Aluno de Mestrado do Programa de Pós-graduação em Patologia da FFFCMPA; ${ }^{4}$ Alunas de Graduação em Medicina da FFFCMPA.

Recebido 16 Junho 2005, recebido na forma final 16 Agosto 2005. Aceito 20 Outubro 2005.

Dra. Arlete Hilbig - Departamento de Ciências Morfológicas / FFFCMPA - Rua Sarmento Leite 245 - 90050-170 Porto Alegre RS Brasil. E-mail: hilbiga@fffcmpa.tche.br 
roepitelial disembrioplástico (DNT) e pineocitoma pode ser difícil.

Não existe, até o momento, um marcador sensível e específico para a célula oligodendroglial e difere ntes tipos de tumores podem mostrar áreas oligodend rogliais. Por outro lado, pode haver expressão de diferentes marcadores em OL típico, por vezes trazendo mais confusão. $O$ diagnóstico diferencial deve preferencialmente ser baseado em dados clínicos (localização do tumor, idade e história clínica), neuroimagem, achados histopatológicos e imunoistoquímica. Entretanto, muitas vezes os dados clínicos podem ser escassos para o patologista, dificultando a diferenciação entre os tumores.

O objetivo do presente estudo foi determinar a expressão de anticorpos rotineiramente utilizados para diferenciação em uma amostra de tumores com características microscópicas de OL.

\section{MÉTODO}

Foram revisados 42 casos de OL diagnosticados pelo exame histopatológico de rotina no Departamento de Patologia da FFFCMPA com amostra tumoral suficiente para o estudo. Todas as lâminas foram revisadas pelos autores para confirmação diagnóstica e posteriormente foi realizada imunoistoquímica, utilizando a técnica da estreptoavidinabiotina-peroxidase, descrita a seguir. Os dados clínicos foram informados pelos neurocirurgiões que encaminharam o material para estudo, pois as amostras eram provenientes de diferentes serviços de neurociru rgia na cidade de Porto Alegre.

Imunoistoquímica - Todo o tecido estudado foi previamente fixado em formalina a $10 \%$ e incluído em parafina. Os blocos foram cortados em micrótomo com $5 \mu \mathrm{m}$ de espessura e montados em lâminas preparadas com org a nocilano. As lâminas foram desparafinadas e processadas para imunoistoquímica, utilizando os anticorpos primários descritos na Tabela, através das seguintes etapas: (1) re cu peração antigênica através de aquecimento em solução de citrato de sódio $(\mathrm{pH}: 6,0)$ - para anticorpos sinaptofisina e NeuN; (2) bloqueio da peroxidase endógena em metanol e H2O2; (3) incubação em soro normal; (4) pernoite em anticorpo primário em refrigerador; (5) anticorpo secundário específico (Novocastra Lab); (6) complexo estreptoavidinabiotina-peroxidase (Novocastra Lab); (7) 3-3' diaminobenzidina/H2O2 e contra-coloração com hematoxilina. Entre cada etapa, o tecido era lavado $3 \mathrm{X}$ em solução tampão fosfato (PBS). Foram utilizados controle positivo e negativo (sem anticorpo primário) em todas as reações.

\section{RESULTADOS}

Os casos estudados apresentavam características típicas de OL: estrutura uniforme de células com núcleo pequeno e redondo, citoplasma claro e membrana celular bem definida, apresentando vasos deli-

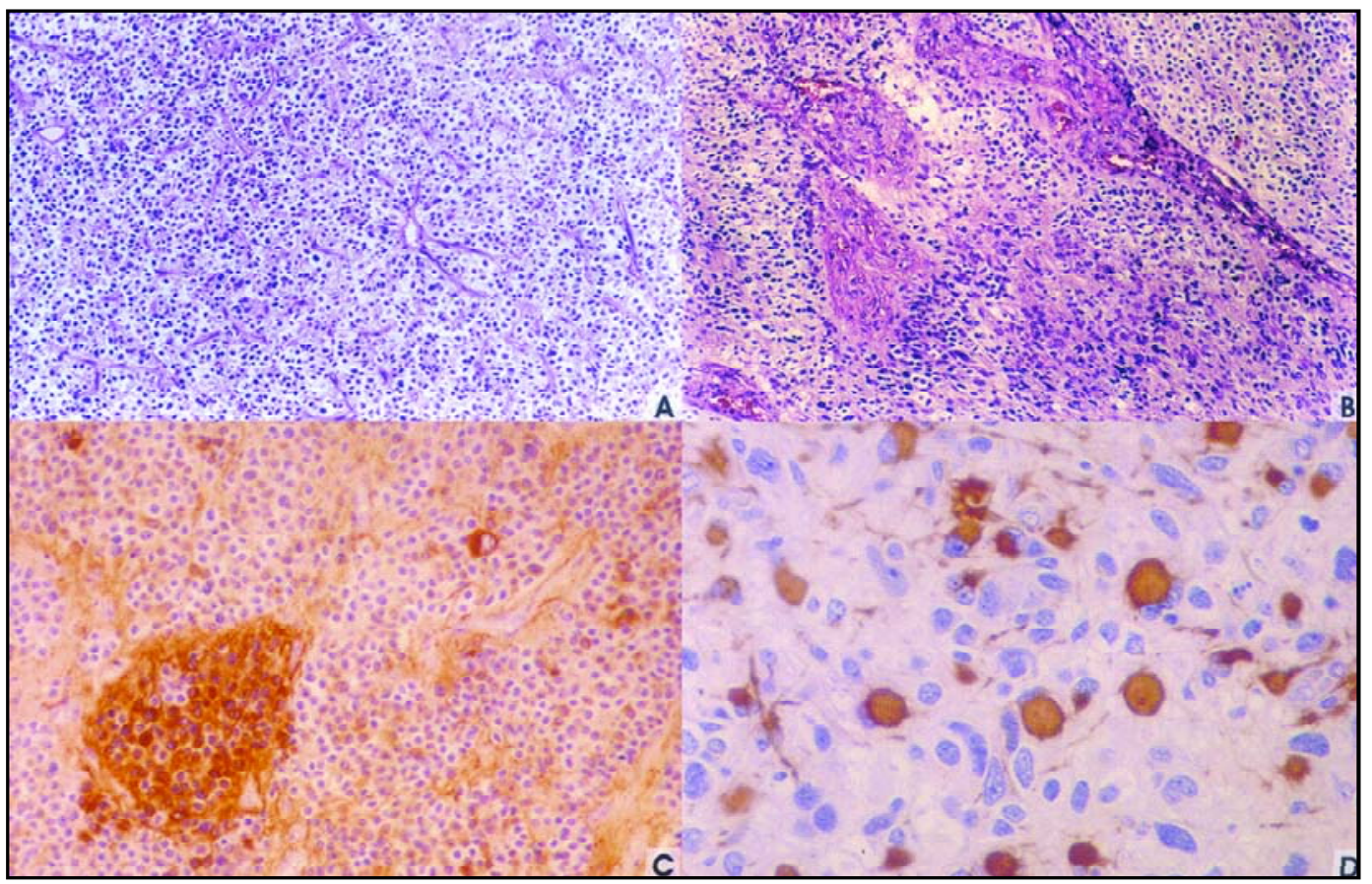

Fig 1. A) Oligodendroglioma apresentando halo claro perinuclear e vasos delicados e ramificados com padrão típico (obj. 20X); B) Oligodendroglioma anaplásico com hiperplasia endotelial e área de necrose (obj. 10X); C) OL com área de positividade para GFAP (obj. 40X); D. Positividade para GFAP em microgemistocitos (obj. $40 X)$. 
Tabela. Imunoistoquímica nos oligodendrogliomas.

\begin{tabular}{|c|c|c|c|c|}
\hline Anticorpo primário & Diluição & Especificidade & $\begin{array}{l}\text { Positivo } \\
\text { (n/total) }\end{array}$ & Focal \\
\hline GFAP (DAKO) & $1 / 800$ & Proteína ácida da fibra glial & $33 / 42$ & $11 / 33$ \\
\hline NSE (DAKO) & $1 / 500$ & $\gamma$ subunidade da enolase & $23 / 42$ & $7 / 23$ \\
\hline Sinaptofisina (DAKO) & $1 / 100^{*}$ & Proteína de membrana da vesícula sináptica - neurônios & $12 / 42$ & $9 / 12$ \\
\hline S-100 Protein & Kit Immustain & Proteína S-100 & $34 / 42$ & $7 / 34$ \\
\hline NeuN (Chemicon) & $1 / 1000 *$ & Proteína nuclear específica de neurônios pós-mitóticos & $2 / 42$ & $2 / 2$ \\
\hline
\end{tabular}

* recuperação antigênica.

cados e ramificados com aspecto de "cerca de arame" (Fig 1A). Dos 42 casos estudados, 10 apresentaram sinais de maior malignidade como áreas de necrose, hiperplasia do endotélio vascular ou aumento do número de mitoses, sendo diagnosticados como OL anaplásico (Fig 1B). Um caso apresentava, além de áreas oligodendrogliais, extensas áreas astrocíticas, sendo diagnosticado como oligoastrocitoma. Em relação à localização, 9 casos $(21,43 \%)$ eram na região frontal; 4 (9,52\%) na região temporal; 4 na região parietal; 2 (4,76\%) estavam localizados em ventrículo lateral; e 1 no corpo caloso. Na maioria (22 casos $52,4 \%)$, a localização exata não foi informada pelo cirurgião. A idade dos pacientes variou de 4 a 60 anos com média de 43,5 anos e desvio padrão de 12,62.

Imunoistoquímica - A expressão de GFAP ocorre u em 33 casos (78,57\%). Seis casos expressaram intensamente GFAP, especialmente em microgemistocitos (Fig 1D). A expressão muitas vezes foi focal (Fig 1C) e em 11 casos houve expressão apenas em poucas células. Doze casos $(28,57 \%)$ apresentaram algum grau de positividade para sinaptofisina, em pequenas áreas em 9 casos (Fig 2A). Consideramos como positivo somente quando a expressão era em áreas centrais do tumor, tendo o cuidado de excluir aqueles com expressão apenas em áreas de infiltração do tecido normal. Em 3 casos (2 localizados em ventrículo lateral e 1 sem localização especificada), houve positividade em extensas áreas do tumor, indicando uma difere nciação neuronal e levando ao diagnóstico de neurocitoma central (Fig 2B). Em 3 casos (1 temporal, 1 fronto-parietal e 1 sem localização especificada) existiam á reas com aspecto de DNT positivas para sinaptofisina. Vinte e três casos $(52,38 \%)$ mostraram algum grau de positividade para NSE (Fig 2C), sendo 7 em pequenas áreas. Apenas 2 casos mostraram positividade focal para NeuN (4,76\%). Trinta e quatro casos $(80,95 \%)$ foram positivos para proteína S-100 (Tabela).

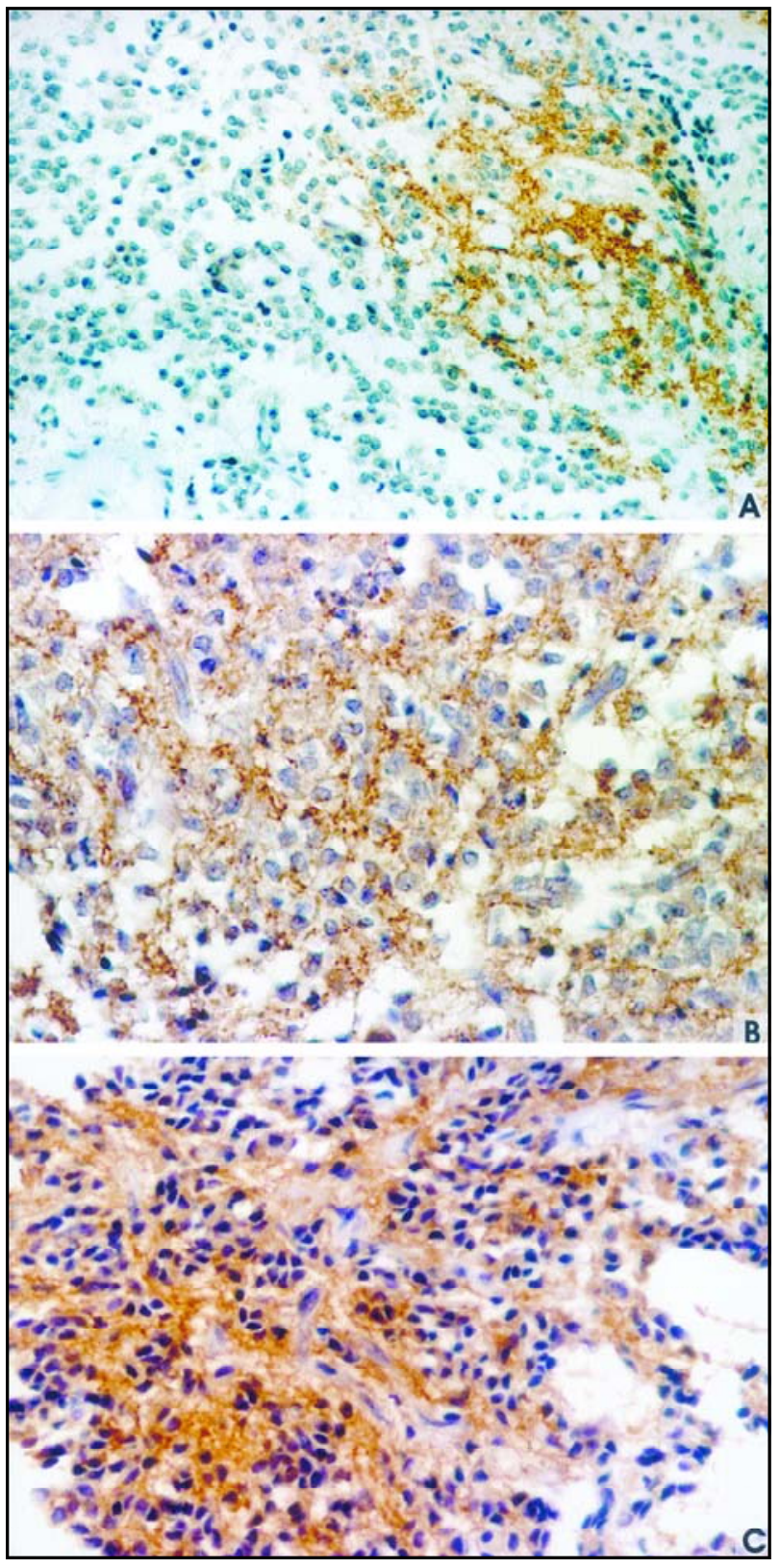

Fig 2. A) Positividade focal para sinaptofisina em OL típico; Positividade difusa em neurocitoma central (obj. 40X): B) sinaptofisina: C) Enolase Neurônio Específica (NSE). 


\section{DISCUSSÃO}

Nos últimos anos, os achados relacionados às alterações genéticas nos OL têm auxiliado no entendimento e também na confirmação diagnóstica desses tumores. Isto após um período de aumento expressivo no diagnóstico pressionado pela possível quimiossenssibilidade dos OL. As perdas alélicas no braço curto do cromossoma 1 e braço longo do cromossoma 19 são consideradas típicas desses tumores ${ }^{3,4}$. Entretando, a avaliação das alterações genéticas ainda não está disponível na rotina da histopatologia, que mantém o diagnóstico histopatológico baseado em suas características típicas à microscopia óptica e, em casos mais difíceis, reco rre na maioria das vezes à imunoistoquímica. Nesse sentido, a presença de células com núcleo redondo e homogêneo e halo claro perinuclear, em amostras fixadas, é uma característica que identifica os OL. Entretanto, essas características podem também estar presentes e devem ser consideradas no diagnóstico diferencial de outros tumores, tais como: neurocitoma central (NC), DNT e ependimomas de células claras (EP). Existem diferenças na apresentação clínica, histopatologia e também no tratamento e prognóstico desses tumores, sendo de grande importância o seu correto diagnóstico ${ }^{6,7}$. Os dados clínicos informados ao patologista nem sempre são completos, sendo necessário ter em mente todas essas possibilidades diante da hipótese de OL.

O NC é neoplasia rara $(0,25$ a $0,5 \%)$ que acomete geralmente adultos jovens e tipicamente se localiza na região do ventrículo lateral, mostra um padrão consistente de diferenciação neuronal e tem bom prognóstico ${ }^{8,9}$. O DNT é neoplasia glioneural caracterizada por sua forte associação com epilepsia, com localização intracortical, participação de linhagens celulares neuronal e glial, e presença freqüente de anormalidades da arquitetura cortical ${ }^{1,7,10}$. Os EP são tumores de células ependimárias que podem ocorrer ao longo do sistema ventricular, sendo mais freqüentes na fossa posterior, no IV ventrículo.

Esses tumores podem apresentar áreas oligodendrogliais, mas também apresentam áreas mais características na histopatologia, que devem ser procuradas mas nem sempre estão presentes. Nos DNT é útil a identificação de áreas císticas com elemento glioneural específico constituído de pequenas células oligodendroglial-like com arranjo colunar ao longo de axônios separados por uma matriz mixóide onde existem neurônios que parecem flutuar. A presença de zonas fibrilares acelulares é uma característica às vezes presente nos NC; enquanto a presença de pseu- dorosetas perivasculares e rosetas ependimárias é característica dos EP. Em situações de maior dificuldade diagnóstica, o estudo imunoistoquímico com marcadores glial e neuronal pode auxiliar no diagnóstico diferencial, mas existem limitações também na sua utilização. Alguns estudos ${ }^{11,12}$ têm demonstrado bons resultados utilizando marc a $\mathrm{d}$ o res em $\mathrm{OL}$ (Olig1 e Olig2), mas esses anticorpos não são disponíveis comercialmente para uso rotineiro. Portanto, não existe um marcador específico para OL e o estudo imunoistoquímico tem sido utilizado principalmente para excluir outros diagnósticos, mais que para estabelecer a natureza oligodendroglial da neoplasia. Entretanto, é necessária muita cautela na avaliação imunoistoquímica em $\mathrm{OL}$, especialmente em amostras pequenas. Nosso estudo, assim como outros autores $^{10,13-15}$, demonstra que OL pode ser positivo para diferentes anticorpos, muitas vezes de forma focal, sendo possível encontrar áreas de diferenciação neuronal e glial em OL típicos.

Na nossa casuística, os OL mostraram áreas de positividade para GFAP em $78 \%$ dos tumores, sendo em poucas células em $1 / 3$ dos casos. Oligodendrócitos normais não expressam GFAP, mas OL típicos e formas mistas, constituídas de uma mistura de astrócitos e oligodendrócitos, podem expressá-lo. Koperek et al. ${ }^{16}$ encontraram positividade para GFAP em todos os 10 OL típicos estudados, sendo parcial na metade dos casos. A expressão de GFAP nos OL pode ser explicada pela presença de alguns astrócitos maduros reativos dentro do tumor ou de formas transicionais entre astrócitos e oligodendrócitos, denominados microgemistocitos. É possível também que as células tumorais positivas representem oligodendrócitos imaturos, que podem expressar transitoriamente GFAP. As formas que apresentam maior grau de malignidade, com hiperplasia endotelial e áreas de necrose, podem ser classificadas por alguns autores como glioblastoma com componente oligodendroglial, e não existem critérios claros para essa diferenciação, ficando muitas vezes à critério do patologista e dependendo da extensão da área oligodendroglial na amostra avaliada, a classificação como OL anaplásico ou glioblastoma. Têm sido sugerido ${ }^{5,6}$ que glioblastomas com áreas oligodendrogliais apresentam melhor prognóstico, e nesses tumores podemos encontrar positividade para marcadores gliais e às vezes neuronais. OL típicos também podem expressar proteína S-100 ${ }^{17}$, o que foi demonstrado em $80 \%$ dos nossos casos, independentemente da expressão ou não de GFAP.

Áreas focais de diferenciação neuronal também podem estar presentes em $\mathrm{OL}$, tendo ocorrido em 
$28,6 \%$ dos casos estudados. A demonstração da p resença de genes com funções relacionadas e abundantemente expressos em neurônios normais em OL com perda $1 q^{15}$, sugere que os OL podem ter características neuronais, apesar de seremprovavelmente originados a partir de células de linhagem glial. Como os OL são tumores infiltrativos e muitas vezes estão localizados próximo ao córtex, deve existir o cuidado para não considerar como positivas as áreas de infiltração do tumor em tecido normal. A expressão intensa para marcadores neuronais, entretanto, deve favorecer o diagnóstico de NC, especialmente se a localização for ventricular, o que ocorreu em três casos estudados. Já é bem conhecido que a expressão de NSE pode ocorrer em tumores gliais ${ }^{18}$, não sendo específica como marcador neuronal. Em nosso estudo, a expressão abundante de sinaptofisina associada a NSE, foi suficiente para demonstrar presença de diferenciação neuronal. Outro marcador neuronal que tem sido utilizado de maneira crescente e está expresso especialmente no núcleo de neurônios mad u ros é o NeuN. Alguns autores ${ }^{10,16,19}$ sugerem que seria o anticorpo de escolha pela sensibilidade e especificidade no diagnóstico de NC. Na nossa casuística ele mostrou-se positivo focalmente em dois casos estudados.

Concluindo, nosso trabalho demonstra que podemos encontrar áreas com expressão de marcador glial (GFAP) ou neuronal (sinaptofisina/NSE/NeuN) em OL típicos. Portanto, é necessária cautela na avaliação imunoistoquímica para diagnóstico diferencial desse tumor, especialmente em amostras pequenas.

\section{REFERÊNCIAS}

1. Lantos PL, Louis M, Rosemblum MK, Kleihus P. Tumours of the nervous system. In Graham DI and Lantos PL (eds). Greenfield's Neuropathology. London: Arnold, 2002.
2. Sanson M, Aguirre-Cruz L, Cartalat-Carel S, Hoang-Xuan K. Oligodendrogliomas: an update on basic and clinical research. Curr Neurol Neurosci Rep 2003;3:223-228.

3. Felsberg J, Erkwoh A, Sabel MC, et al. Oligodendroglial tumors: refinement of candidate regions on chromosome arm $1 \mathrm{p}$ and correlation of 1p/19q status with survival. Brain Pathol 2004;14:121-130.

4. Chahlavi A, Kanner A, Peereboom D, Staugatis SM, Elson P, Barnett G. Impact of chromosome 1p status in response of oligodendroglioma to temozolomide: preliminary results. J Neurooncol 2003;61:267-273.

5. Pinto LW, Chimelli L. Componente oligodendroglial e neuronal em glioblastoma: possível relação com prognóstico. A rq Neuropsiquiatr 2004;62:1074-1078

6. Kleihues P, Louis DN, Scheithauer BW, et al. The WHO classification of tumors of the nervous system. J Neuropathol Exp Neurol 2002;61:215-225.

7. McLendon RE, Provenzale J. Glioneuronal tumors of the central nervous system. Brain Tumor Pathol 2002;19:51-58.

8. Kim DG, Chi JG, Park SH, et al. Intraventricular neurocytoma: clinicopathological analysis of seven cases. J Neurosurg 1992;76: 759-765.

9. Kubota T, Hayashi M, Kawano H, et al. Central neurocytoma: immunohistochemical and ultrastructural study. Acta Neuropath ol 1991;81: 418-427.

10. Wolf HK, Buslei R, Blümcke I, Wiestler OD, Pietsch T. Neuronal antigens in oligodendrogliomas and dysembryoplastic neuroepithelial tumors. Acta Neuropathol 1997;94:436-443.

11. Yokoo H, Nobusawa S, Isoda K, et al. Establishment of anti-human Olig2 antibody and successful application to detect human oligodendrocytes and oligodendroglial tumors on formalin-fixed, paraffin-embedded tissue sections. Brain Pathol 2003; XV th International Congress of Neuropathology. Torino-Lingotto, September 14-18:145.

12. Azzarelli B, Miravalle L, Vidal R. Immunolocalization of oligodendrocyte transcription factor 1 (Olig1) in brain tumors. J Neuropathol Exp Neurol 2004;63:170-179.

13. Perry A, Scheithauer BW, Macaulay RJ, Raffel C, Roth KA, Kros JM. Oligodendrgliomas with neurocytic diff e rentiation: a report of four cases with diagnostic and histogenetic implications. J Neuropathol Exp Neurol 2002;61:947-955

14. Wharton SB, Chan KK, Hamilton FA, Anderson JR. Expression of neuronal markers in oligodendrogliomas: an immunohistochemical study. Neuropathol Appl Neurobiol 1998;24:302-308.

15. Mukasa A, Ueki K, Ge X, et al. Selective expression of a subset of neuronal genes in oligodendroglioma with chromosome 1p loss. Brain Pathol 2004; $14: 34-42$

16. Koperek O, Gelpi E, Birner P, et al. Value and limits of immunohistochemstry in diff e rential diagnosis of clear cell primary brain tumors. Acta Neropathol (Berlin) 2004;108:24-30.

17. Nakopoulou L, Kerekoudi E, Thomaides T, Litsios B. An immunocytochemical comparison of glial fibrillary acidic protein, S-100 and vimentin in human glial tumors. J Neurooncol 1990;8:33-40.

18. Vinores SA, Bonnin JM, Rubinstein LJ, Marangos PJ. Immunohistochemical demonstration of neuron-specific enolase in neoplasm of the CNS and other tissues. Arch Pathol Lab Med 1984; 108:536-540.

19. Soylemezoglu F, Onder S, Trezel GG, Berker M. Neuronal nuclear antigen (NeuN): a new tool in the diagnosis of central neurocytoma. Pathol Res Pract 2003;199:463-468. 